\title{
White adipose tissue coloring by intermittent fasting
}

\author{
Cell Research (2017) 27:1300-1301. doi:10.1038/cr.2017.130; published online 24 October 2017
}

\begin{abstract}
Intermittent fasting (IF) has been shown to promote metabolic health in several organisms. Two recent papers show that IF induces white adipose tissue beiging and increases thermogenesis, which improves metabolic health in mice.
\end{abstract}

The incidence of obesity and type 2 diabetes is sharply increasing globally. White adipose tissue (WAT) serves primarily as an energy storage depot during overnutrition, but emerging evidence suggests that WAT can be converted towards energy-using tissue, when white adipocytes acquire features of beige/brown adipocytes. Rather than storing energy, these cells then start to use extra energy for heat production, which could be a promising therapeutic approach to treat obesity-related metabolic disorders. In a study recently published by Cell Research, Kim et al. [1] demonstrate that IF increases vascular endothelial growth factor (VEGF) levels in WAT, which induced angiogenesis, adipose tissue macrophage polarization and WAT browning/beiging. This led to improved insulin sensitivity, which counteracted obesity, and alleviated metabolic syndrome in the mice. Another mechanism mediating the effects of IF was recently proposed by Li et al. [2], who showed that IF promotes WAT browning via modulating gut microbiota and providing its fermentation products acetate and lactate.

In mice, VEGF-induced angiogenesis in adipose tissue has been shown to increase thermogenesis and decrease tissue inflammation, counteracting obesity and insulin resistance induced by diet $[3,4]$. However, targeted delivery of VEGF to adipose tissue is challeng- ing, as systemic delivery of VEGF leads to vascular leakiness in several organs. VEGF-VEGFR2 pathway activation is induced indirectly by VEGF-B, which displaces VEGF from VEGFR1. An excess of VEGF-B was recently shown to result in increased adipose vasculature, beiging of WAT and increased energy expenditure, leading to improved metabolic health in mice [5]. These effects of VEGF-B were limited by the endogenous VEGF levels; thus, it appears to be a safer approach than VEGF delivery for VEGFR2 activation in vivo. However, it is still unclear, how activation of VEGFR2 in WAT could be achieved for the development of effective and safe treatment for humans. Kim et al. [1] demonstrate that by allowing mice to fast every three days, VEGF levels were significantly increased in WAT. This was associated with angiogenesis, macrophage polarization towards antiinflammatory M2 type, and beiging of the WAT. The importance of adipocytederived VEGF was demonstrated using adipocyte-specific VEGF-deficient mice (aP2-Cre; Vegfa $\left.a^{f o x f f o x}\right)$, which did not show any of the abovementioned changes in WAT or improvement in whole body metabolism.

An almost simultaneously published paper in Cell Metabolism reported a similar WAT beiging phenotype and reversal of high-fat-diet-induced obesity and metabolic disorders using a similar diet regimen (this case every-other-day fasting) [2]. In this study, IF was shown to increase small intestine length and to affect the composition of gut microbiota. The importance of gut microbiota was demonstrated by fecal transplantation from the IF-treated and control mice to microbiota-depleted (antibiotic-treated) mice. The WAT beiging effect was induced by microbiota transplantation from IF-treated mice into microbiotadepleted mice, whereas the IF effects on WAT were absent in the microbiotadepleted mice. Li et al. suggest that the beiging stimulus was mediated by the fermentation products acetate and lactate, which were strongly increased in the gut and serum after IF. In addition, monocarboxylate transporter 1 (Mct1) mRNA was increased several fold in adipose tissue, suggesting that the uptake of monocarboxylates (e.g., lactate) into adipocytes was increased.

These data show that in mice, IF induces beiging of subcutaneous WAT, which increases thermogenesis and improves metabolic health upon dietinduced obesity. Interestingly, the effects seem to be specific for the beiging of subcutaneous WAT as BAT activation was not observed. The study by Kim et al. provides evidence for the importance of adipose tissue VEGF, while Li et al. demonstrate the essential role of microbiota in the IF-induced effects (Figure 1). These findings raise several interesting questions. First, are the mechanisms presented in these two studies connected? For example, could the increased acetate or lactate induce VEGF production by adipocytes? Does the increased vasculature contribute essential angiocrine signals from activated endothelial cells to adipocytes? Could macrophage polarization be induced in adipose tissue by other non-invasive means that could be applied to humans? Importantly, the IF program used in these studies could in principle be applied to humans. In fact, some religious 


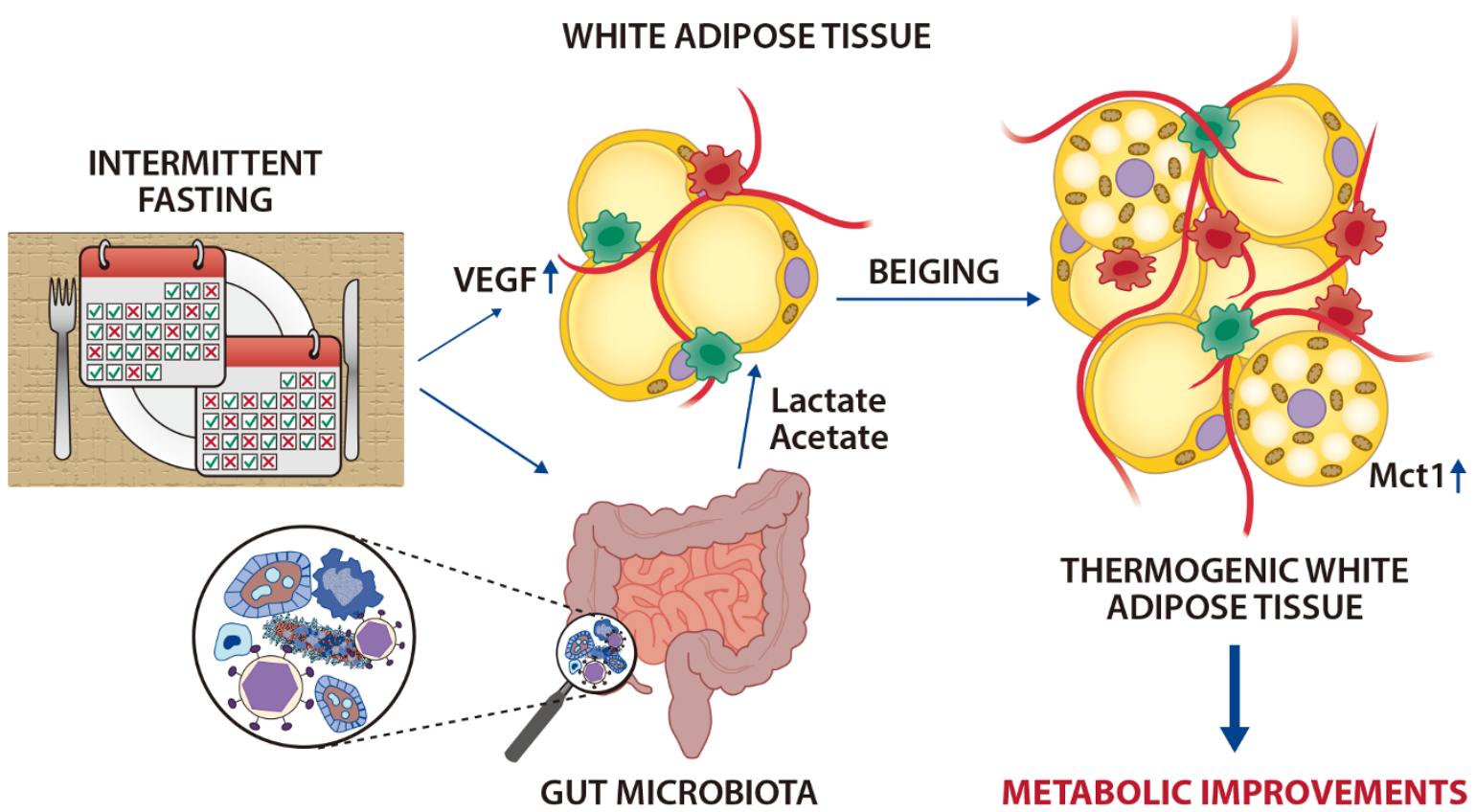

Figure 1 Intermittent fasting (IF) induces beiging of the white adipose tissue (WAT) and increases thermogenesis and metabolic health in mice. Two recent studies reported similar beiging effect of WAT by IF. The study by Kim et al. demonstrated the importance of increased VEGF production and macrophage activation in WAT, which was also accompanied by increased vasculature in WAT. The other study by Li et al. provided evidence that IF induced marked changes in gut microbiota, which are essential for the beiging of the WAT via production of the fermentation products lactate and acetate. In both studies, IF was shown to have beneficial effects on body weight and whole body metabolic health in mice (green cells = M1 macrophages, red cells = M2 macrophages).

communities already adhere to certain types of intermittent fasting regimens. However, rigorous adherence to fasting in the fast-growing population of obese and overweight patients would be unrealistic. It would be important to find out whether less stringent restrictions of dietary habits would result in similar effects that are beneficial also in humans.

\section{Riikka Kivelä $^{1}$, Kari Alitalo ${ }^{1}$}

${ }^{\prime}$ Wihuri Research Institute and Translational Cancer Biology Program, Faculty of Medicine, University of Helsinki, Helsinki, Finland Correspondence: Riikka Kiveläa, Kari Alitalo ${ }^{\mathrm{b}}$

a'E-mail: riikka.kivela@helsinki.fi

bE-mail: kari.alitalo@helsinki.fi

\section{References}

1 Kim K-H, Kim YH, Son JE, et al. Cell Res 2017; 27:1309-1326.

2 Li G, Xie C, Lu S, et al. Cell Metab 2017; 26:672-685.

3 Cao Y. Cell Metab 2013; 18:478-489.

4 Sung HK, Doh KO, Son JE, et al. Cell Metab 2013; 17:61-72.

5 Robciuc MR, Kivela R, Williams IM, et al. Cell Metab 2016; 23:712-724. 